\title{
Protocolos e iniciativas de atendimento médico-legal em casos de violência sexual em mulheres: comparação entre os achados no Brasil e no mundo
}

\author{
Protocols and initiatives for medico-legal assistance in sexual \\ assaults cases against women: a comparison of initiatives in \\ Brazil and abroad
}

\author{
Fabianne Ribeiro Bonnet ${ }^{1}$; Raquel Barbosa Cintra ${ }^{2}$
}

\begin{abstract}
Bonnet FR; Cintra RB. Protocolos e iniciativas de atendimento médico-legal em casos de violência sexual em mulheres: comparação entre os achados no Brasil e no mundo. Saúde, Ética \& Justiça. 2014;19(1);45-51.

RESUMO: Estudos recentes sobre violência sexual, particularmente sobre crimes e agressões praticadas contra mulheres, apontam para a elevada incidência do fenômeno, que é de caráter transversal em todos os países do mundo, culturas e estratos sociais e econômicos. Tais estudos revelam, ainda, que as taxas de acusação e de condenação nos casos de crimes sexuais em geral são baixas. Este problema tem sido uma fonte recorrente de frustrações para as vítimas sobreviventes, advogados das vítimas, bem como para os membros do sistema jurídico-penal de países em todo o mundo. Nesse contexto, a partir de uma leitura crítica de um conjunto de estudos científicos, documentos normativos e práticas selecionadas de países de cinco continentes, este trabalho objetiva comparar protocolos e iniciativas de atendimento médico-legal em casos de violência sexual contra mulheres, identificando as principais lacunas e desafios no Brasil. Dessa forma, poderão ser ampliadas as possibilidades de identificação e condenação de agressores, na perspectiva de inibição de novos crimes desta natureza.
\end{abstract}

DESCRITORES: Violência Sexual; Medicina Legal; Protocolos; Brasil; Estratégias Nacionais; Regionalização.

\footnotetext{
Pós-graduanda do curso de especialização em Medicina Legal e Perícias Médicas do Departamento de Medicina Legal, Ética Médica e Medicina Social e do Trabalho da Faculdade de Medicina da Universidade de São Paulo.

2 Universidade de Moji das Cruzes, UMC.

Endereço para correspondência: Fabianne Ribeiro Bonnet. Avenida Doutor Arnaldo, 455, Departamento de Medicina Legal, Ética Médica e Medicina Social e do Trabalho da Faculdade de Medicina da Universidade de São Paulo. Cerqueira César - São Paulo, SP. CEP 012903-000. E-mail: fabianne.trab@gmail.com
} 


\section{INTRODUÇÃO}

A violência sexual é um crime que ocorre indistintamente em todas as regiões do mundo, podendo atingir tanto homens quanto mulheres. Quando ocorre com mulheres, atinge todas as faixas etárias, etnias, aparências, estados civis, classes sociais, culturas, orientações sexuais e estados de saúde ${ }^{1,2}$. Em um conceito bem amplo, caracteriza-se como a utilização da força física ou intimidação para a obtenção de contato sexual sem o consentimento da vítima ${ }^{3}$. Cada país conta com uma definição própria, legislação diferenciada e específica sobre o tema, bem como diversas penalidades e jurisprudências. No Brasil, o crime de estupro é definido através do Artigo 213 do Código Penal ${ }^{4}$, cuja revisão mais recente é dada pela Lei 12.015, datada de 20095: "Constranger alguém, mediante violência ou grave ameaça, a ter conjunção carnal ou a praticar ou permitir que com ele se pratique outro ato libidinoso".

Acredita-se que a violência sexual seja um problema de amplitude crescente, porém de difícil mensuração, pois os dados estatísticos não refletem a realidade, por conta da subnotificação ${ }^{1,2,3}$. As vítimas, seja por vergonha, medo, ameaças, constrangimentos diversos, podendo incluir um processo de acolhimento com falta de sensibilização em algumas delegacias e serviços médico-legais, até descrenças na condenação dos agressores, que muitas vezes são familiares ou conhecidos, via de regra, não procuram os órgãos competentes para realizar a denúncia ${ }^{1,2}$. Entretanto, os impactos e reflexos desse tipo de crime, a curto e longo prazo, podem ser caracterizados como um problema de saúde pública, já que implicam em danos físicos e psicológicos, dificuldade de reinserção na sociedade, abuso de substâncias como álcool e drogas, altos índices de suicídio, sem deixar de mencionar as doenças sexualmente transmissíveis e a gravidez indesejada ${ }^{1,2}$.

No caso particular do Brasil, a crescente implementação de políticas públicas visando ao combate à violência contra a mulher, incluindo a publicação da Lei $\mathrm{n}^{\circ} 12.845$ de 01 de agosto de $2013^{6}$, que institui o atendimento integrado das vítimas de violência sexual, conta, por exemplo, com a criação das Delegacias Especializadas $^{7}$, que se propõem a oferecer um serviço diferenciado para que a sobrevivente se sinta mais confiante e confortável para realizar as denúncias e, assim, aumentar a chance de condenação dos agressores. Contudo, além dos ganhos ainda serem escassos frente à demanda existente ${ }^{7}$, a identificação dos suspeitos, pré-requisito para a possibilidade de condenação, fica condicionada à existência de provas obtidas através de práticas periciais e coleta de amostras, estabelecidas e padronizadas sob a forma de protocolos e conduzidas por profissionais especializados e em instalações igualmente adequadas.

Atualmente, diversos países já fazem uso de protocolos próprios, o que incentivou o desenvolvimento de um trabalho de pesquisa de comparação entre protocolos e iniciativas de atendimento de casos de violência sexual que, futuramente, possa contribuir para a eliminação das lacunas existentes no País nesse campo e levar a uma padronização de todo o processo pericial em casos de violência sexual em mulheres. Desta forma, maiores serão as possibilidades de identificação e condenação de agressores, podendo também servir para inibição de novos crimes desta natureza.

\section{OBJETIVOS}

Comparar protocolos e iniciativas de atendimento médico-legal em casos de violência sexual em mulheres em países dos cinco continentes, identificando as principais lacunas e desafios no Brasil.

\section{MÉTODOS}

Segundo a taxonomia proposta por Vergara ${ }^{8}$, a pesquisa pode ser classificada, quanto à abordagem, como qualitativa; quanto à natureza, aplicada e quanto aos fins, descritiva. Com relação aos meios de investigação, utilizaram-se os seguintes métodos em seu desenvolvimento: pesquisa bibliográfica, pesquisa documental e análise de conteúdo de trabalhos científicos, documentos normativos e legais relacionados à questão principal da pesquisa.

A pesquisa bibliográfica foi realizada mediante consulta direta às seguintes bases de dados: Web of Science; Scopus; Scielo, Lilacs e MedLine. A escolha dessas bases de dados foi devida a diferenças de abrangência, à qualidade de indexação dos trabalhos e à existência de ferramentas estatísticas que facilitaram a seleção da informação de interesse para a pesquisa (por exemplo, filtro dos trabalhos mais citados). A estratégia de busca incluiu os seguintes termos: "sexual violence"; "sexual assault"; "rape"; "medico-legal evidence"; "medico-legal intervention"; "forensic evidence"; bem como os operadores booleanos (AND, OR e NOT) disponibilizados pelas bases Web of Science e Scopus. Os resultados obtidos de cada base foram organizados em planilha do Microsoft Excel巴, para facilitar a comparação, classificar as referências por subtema e excluir as duplicatas, como preparação para a etapa de análise de conteúdo propriamente dita.

Em complementação à pesquisa bibliográfica nas bases mencionadas, utilizou-se a ferramenta de busca "Google Scholar" na internet, que resultou na seleção de documentos normativos e relatórios de grande 
interesse para a pesquisa. Finalmente, investigações diretamente em sites de instituições, como a World Health Organization (WHO) e o United States (US) Department of Justice, foram realizadas no sentido de checar ou atualizar informações reportadas nos documentos selecionados.

Para análise das informações e dados secundários coletados, adotou-se o método de análise de conteúdo ${ }^{9}$, categorizando-se os conteúdos dos protocolos, das boas práticas e dos métodos médico-legais apresentados nos documentos e artigos selecionados na fase da revisão bibliográfica.

\section{RESULTADOS E DISCUSSÃO}

Buscou-se sistematizar as boas práticas e os pontos de atenção no atendimento médico-legal em casos de violência sexual através da análise comparativa de protocolos e iniciativas de países de cinco continentes. Os países foram escolhidos como objeto do trabalho levando-se em conta os seguintes critérios:

- diferentes níveis de desenvolvimento econômico-social, cotejando-se a situação daqueles classificados como desenvolvidos (EUA, Inglaterra e países nórdicos) com a dos emergentes (Índia e África do Sul) e a de outros menos favorecidos (países do Leste Europeu);

- alta densidade populacional (Índia)

- grande extensão territorial (Índia e Austrália).

Os protocolos e demais documentos analisados evidenciam muitos pontos de convergência na conduta adotada pelos diversos países para tratar do problema dos crimes de natureza sexual. Os fatores de risco e de sucesso identificados são muito semelhantes, mesmo levando-se em consideração tratar-se de países de diferentes continentes, idiomas, religiões e desenvolvimento socioeconômico.

A adoção de boas práticas em todos os níveis do processo leva a um atendimento de qualidade às sobreviventes de agressão sexual, sendo apontada como fator de sucesso para que sejam consagradas as estratégias traçadas pelos diversos países integrantes da amostra pesquisada. Assim, a equipe médicoforense deve receber treinamento especial nas seguintes situações: (i) ao fazer uso de kits de diagnóstico de qualidade, devidamente acompanhados de folhetos explicativos quanto ao seu manuseio e aplicações; (ii) ao coletar, embalar, identificar, lacrar e estocar corretamente as amostras, sejam elas compostas por fluidos corporais, fios de cabelo, recortes de unhas etc., seguir manuais de orientações específicas, dando condições para que a amostra obtida se transforme em uma evidência concreta, a ser utilizada em um processo criminal bem sucedido. Da mesma forma, as boas práticas devem nortear a elaboração de relatórios médicos e histórico do examinado, todos devidamente preenchidos com as informações relevantes sobre o ocorrido e assinados pelos examinadores ${ }^{2,10,11}$.

Por outro lado, é destacado como um fator de atenção a necessidade desses centros de atendimento estarem acessíveis aos sobreviventes de um caso de agressão, o que se sabe ser particularmente difícil em países com dimensões quase continentais como a Índia, Austrália, Estados Unidos e Brasil. Longos deslocamentos para chegar até eles ou horas de espera para passar pelo exame da equipe médico-forense representam um desestímulo ${ }^{2,11-13}$. Os serviços de aconselhamento jurídico também devem ser oferecidos de imediato, no mesmo local do atendimento médico, sob pena de as vítimas também desistirem de dar continuidade a uma ação judicial ${ }^{11,13}$.

Na Tabela 1 a seguir, através da identificação de 14 itens considerados relevantes para os propósitos do presente trabalho, são evidenciadas: as boas práticas do atendimento médico-legal nos casos de violência sexual, os países estudados que já as seguem e a realidade brasileira.

A identificação de agressores e a sua consequente condenação pelo sistema judiciário estão intimamente ligadas à acurácia do processo pericial médico-legal. Para atingir esse estágio, além de se dispor de Protocolos bem estruturados, é necessário investir em treinamento, a exemplo dos Estados Unidos e Inglaterra.

Conhecer em detalhes a experiência externa pode ser de grande valor, no sentido de queimar etapas, entre elas a que tange ao treinamento de pessoal. $\mathrm{O}$ estabelecimento de convênios de cooperação e visitas técnicas, intercâmbios etc. são soluções de custo relativamente baixo e de alto retorno em termos de aprendizado e disseminação de conhecimentos.

Considerando que Índia e Brasil compartilham estágio similar de mercado emergente (BRICS), que ambos possuem grande extensão territorial e densidade populacional e que a Índia já dispõe de um manual detalhado ("Manual for Medical Examination of Sexual Assault") para abordagem de casos de violência sexual, sua experiência poderia ser utilizada como modelo inicial.

Para contextualizar em termos quantitativos a realidade brasileira atual, a tabela abaixo (Tabela 2) apresenta a frequência de notificações por estupro, a partir de 2010, já ao amparo da Lei 12.015/20095 adiante apresentada, cuja redação tornou mais abrangente a definição de estupro, nela incluindo todos os atos de natureza sexual. 
Bonnet FR; Cintra RB. Protocolos e iniciativas de atendimento médico-legal em casos de violência sexual em mulheres.

TABELA 1 - Atendimento médico-legal em casos de violência sexual em mulheres: boas práticas aplicáveis, países onde são adotadas e realidade brasileira

\begin{tabular}{|c|c|c|}
\hline Item & Boas práticas aplicáveis ao caso brasileiro e países onde são adotadas & Realidade brasileira \\
\hline $\begin{array}{l}\text { Instalação e área } \\
\text { física }\end{array}$ & $\begin{array}{l}\text { Disponíveis sob a forma de centros integrados de atendimento, onde as sobreviventes } \\
\text { são acolhidas em salas que garantam sua privacidade. } \\
\text { Reino Unido e EUA. } \\
\text { África do Sul, Austrália, Índia e República Tcheca: contam com poucos centros. }\end{array}$ & $\begin{array}{l}\text { Restrito às instalações } \\
\text { existentes nos Institutos } \\
\text { Médico-Legais (IML), à } \\
\text { exceção do Hospital Perola } \\
\text { Byington. }\end{array}$ \\
\hline $\begin{array}{l}\text { Equipamentos e } \\
\text { instrumental }\end{array}$ & $\begin{array}{l}\text { Salas de exame equipadas minimamente com colposcópios com armazenamento de } \\
\text { imagens, microscópio e anuscópio. } \\
\text { Reino Unido, EUA, Índia e Austrália. }\end{array}$ & Sem padronização. \\
\hline $\begin{array}{l}\text { Recursos humanos } \\
\text { especializados em } \\
\text { Medicina Legal }\end{array}$ & $\begin{array}{l}\text { Existência de programa de incentivos com estabelecimento de staffs mínimos compostos } \\
\text { por médicos e enfermeiras forenses. } \\
\text { Reino Unido e EUA. } \\
\text { África do Sul, Índia, Romênia e Austrália: equipes treinadas, porém não especializadas } \\
\text { e ainda em número reduzido. }\end{array}$ & $\begin{array}{l}\text { Escassos em face da grande } \\
\text { demanda. Existência } \\
\text { de apenas um curso } \\
\text { de residência médica } \\
\text { especializado em todo o país } \\
\text { e poucas pós-graduações. }\end{array}$ \\
\hline $\begin{array}{l}\text { Sensibilização e } \\
\text { capacitação para os } \\
\text { profissionais de saúde }\end{array}$ & $\begin{array}{l}\text { Campanhas de sensibilização, treinamento permanente para capacitação e com } \\
\text { exigência de renovação da certificação periodicamente. } \\
\text { Reino Unido e EUA. } \\
\text { Malta: em apenas uma região. }\end{array}$ & $\begin{array}{l}\text { Necessidades identificadas } \\
\text { na Norma Técnica do } \\
\text { Ministério da Saúde (2005) } \\
\text { e no Decreto } 7.958 / 2013 \text {. Na } \\
\text { prática, porém, as iniciativas } \\
\text { estão concentradas no estado } \\
\text { de São Paulo. }\end{array}$ \\
\hline
\end{tabular}

\begin{tabular}{|c|c|c|}
\hline $\begin{array}{l}\text { Existência de } \\
\text { Protocolo de } \\
\text { Atendimento Médico- } \\
\text { legal }\end{array}$ & $\begin{array}{l}\text { Disponíveis na amostra de países pesquisados, padronizando e detalhando todos os } \\
\text { procedimentos envolvidos. } \\
\text { Reino Unido, EUA, Índia e África do Sul. } \\
\text { Malta: em apenas uma região. } \\
\text { Austrália: em poucos locais e não é seguido. }\end{array}$ & Iniciativas isoladas. \\
\hline $\begin{array}{l}\text { Kit de coleta de } \\
\text { material para } \\
\text { evidências forenses }\end{array}$ & $\begin{array}{l}\text { Sempre disponíveis. Alguns centros de atendimento desenvolvem seus próprios kits. } \\
\text { Reino Unido, EUA, Índia e África do Sul. } \\
\text { Austrália: há kits padronizados em alguns locais, porém geralmente estão em falta. }\end{array}$ & Não há kit padronizado. \\
\hline $\begin{array}{l}\text { Tempo para a } \\
\text { coleta de evidências } \\
\text { forenses }\end{array}$ & $\begin{array}{l}\text { Desejável: em média até uma hora após a chegada da sobrevivente ao centro de } \\
\text { atendimento. } \\
\text { Reino Unido. } \\
\text { EUA e Índia: preconizam o menor tempo possível, porém não especificam quanto. }\end{array}$ & Não há. \\
\hline Cadeia de custódia & $\begin{array}{l}\text { Perfeitamente caracterizada através de etiquetas e códigos de barras padronizados. } \\
\text { Reino Unido, EUA, Índia, África do Sul e Malta. }\end{array}$ & Sim \\
\hline $\begin{array}{l}\text { Consentimento da } \\
\text { sobrevivente }\end{array}$ & $\begin{array}{l}\text { Exigência de consentimento expresso em formulário próprio, através da assinatura da } \\
\text { sobrevivente, do perito forense e de testemunha neutra. } \\
\text { Todos, porém a Índia é o único a ter o respaldo da testemunha neutra. }\end{array}$ & Obtido em caráter verbal. \\
\hline $\begin{array}{l}\text { Relatório detalhado } \\
\text { do(s) delito(s) } \\
\text { sexual(is) alegado(s) }\end{array}$ & $\begin{array}{l}\text { Realizado através de modelo padronizado e assinado pelo perito médico forense } \\
\text { responsável. } \\
\text { Reino Unido, EUA, Índia e África do Sul. }\end{array}$ & Sim. \\
\hline Exame físico geral & $\begin{array}{l}\text { Sempre. } \\
\text { Todos. }\end{array}$ & Sempre. \\
\hline Exame genito-anal & $\begin{array}{l}\text { Sempre realizado. } \\
\text { Todos. }\end{array}$ & Sempre realizado. \\
\hline Exames específicos & $\begin{array}{l}\text { De acordo com a necessidade. } \\
\text { Todos. }\end{array}$ & De acordo com a necessidade. \\
\hline $\begin{array}{l}\text { Aconselhamento e } \\
\text { assistência jurídica à } \\
\text { vítima }\end{array}$ & $\begin{array}{l}\text { Disponíveis desde o início do atendimento da sobrevivente nos centros de atenção. } \\
\text { Reino Unido, EUA, Índia e África do Sul. } \\
\text { Austrália e República Tcheca: poucos locais. } \\
\text { Malta: apenas através de ONGs e em poucos locais. } \\
\text { Romênia: conta apenas com assistência jurídica. }\end{array}$ & $\begin{array}{l}\text { Não há, em caráter } \\
\text { institucional. }\end{array}$ \\
\hline
\end{tabular}

Fonte: CEHAT, 2012 ${ }^{10}$; CONNSACS, 2013 ${ }^{14}$; COSAI, 2013 ${ }^{15}$; Du Mont e White, 2007²; Du Mont e White, 2013 ${ }^{16}$; Higa et al., 2008 ${ }^{19}$; Hospital Pérola Byington, 2013 ${ }^{20}$; NSW Department of Health - Report 1, $2013^{12}$; NSW Department of Health - Report 2, 2013 ${ }^{13}$; South Africa, $2013^{17}$; U.S. Department of Justice, $2013^{18}$; WHO, $2003^{11}$. 
TABELA 2 - Frequência de notificações do Sistema de Informação de Agravos de Notificação por estupro segundo UF de notificação. Período: 2010-2013(*)

\begin{tabular}{ccccc}
\hline Ano & São Paulo & $\begin{array}{c}\text { Rio de } \\
\text { Janeiro }\end{array}$ & $\begin{array}{c}\text { Demais } \\
\text { estados }\end{array}$ & Total \\
\hline 2010 & 4862 & 1092 & 11298 & 17252 \\
\hline 2011 & 7130 & 2389 & 17246 & 26765 \\
\hline 2012 & 9387 & 4511 & 24288 & 38186 \\
\hline $2013\left(^{*}\right)$ & 4183 & 1348 & 10700 & 16231 \\
\hline
\end{tabular}

Fonte: SINAN - Sistema de Informação de Agravos de Notificação - Ministério da Saúde / SVS 21 .

(*) dados parciais até 29/07/2013.

A análise da tabela permite concluir que houve um aumento significativo das notificações pela Área de Saúde, e que o Estado de São Paulo lidera, com uma participação média em torno de $26 \%{ }^{21}$. Verificando-se as estatísticas de estupro divulgadas pela Secretária de Segurança Pública do Estado de São Paulo, para o mesmo período, foram registradas 9.879 ocorrências policiais, em $2010 ; 10.399$, em $2011 ; 12.886$ em 2012 e 3.356 , no $1^{\mathrm{o}}$ trimestre de $2013^{22}$.

Até o presente momento, não há um protocolo nacional ou estadual de atendimento médico-legal. Além da Lei $\mathrm{n}^{\mathrm{o}} 12.845^{6}$ de 2013 supracitada, existem duas publicações de relevância, ainda que de âmbito predominantemente assistencialista: (i) a Norma Técnica de Prevenção e Tratamento dos Agravos Resultantes da Violência Sexual contra Mulheres e Adolescentes, do Ministério da Saúde (2005) ${ }^{23}$ e o (ii) Decreto $n^{\circ} 7.958^{24}$, publicado em março de 2013, que estabelece diretrizes para o atendimento humanizado às vítimas de violência sexual pelos profissionais de segurança pública e da rede de atendimento do Sistema Único de Saúde, e as competências do Ministério da Justiça e do Ministério da Saúde para sua implementação.

$\mathrm{O}$ atendimento especializado à mulher vítima de violência sexual é feito no Estado de São Paulo. Um exemplo bem sucedido das iniciativas em São Paulo é o Projeto "Bem-Me-Quer", implantado já há doze anos ${ }^{25} \mathrm{e}$ que conta com uma unidade do Instituto Médico Legal (IML) especializada dentro do Hospital Pérola Byington - Centro de Referência da Saúde da Mulher ${ }^{25}$. Existe um banco de dados com 3.000 amostras de esperma recolhidas de mulheres e crianças de até 14 anos de idade vítimas de abusos sexuais ${ }^{26}$. Têm-se, inclusive, notícias de que estão sendo desenvolvidos estudos por parte da Secretária de Justiça do Estado, de que o material coletado no projeto Bem-Me-Quer, bem como o banco de dados gerado, possa ser utilizado como ferramenta de trabalho pelo Ministério Público como instrumento de prova ou confrontação com o material genético colhido de supostos autores de crimes sexuais ${ }^{26}$.
A assistência atualmente oferecida no Hospital Pérola Byington engloba, além da prevenção da gravidez decorrente da violência sexual, a realização de abortos previstos em lei; a assistência psicológica e social; a prevenção da hepatite $\mathrm{B}$, da infecção pelo HIV e de doenças sexualmente transmissíveis e tratamento de traumatismos genitais ${ }^{25}$. Além do Pérola Byington, que conta com atendimento assistencial e pericial, já integram a Rede de Atendimento à Mulher, o Centro de Atenção Integral à Saúde da Mulher (CAISM) da Unicamp, o Hospital Geral de Cotia e o Hospital Geral de Itapecerica da Serra, porém estes quatro têm apenas cunho assistencial ${ }^{25}$.

Em Campinas, foi desenvolvido o Protocolo de Enfermagem na Assistência às Mulheres Vítimas de Violência Sexual do CAISM/Unicamp, descrevendo o atendimento imediato e tardio, o acompanhamento ambulatorial e as ações relacionadas à interrupção legal da gravidez decorrente do estupro ${ }^{19}$. Essa iniciativa do CAISM/Unicamp segue a estratégia da utilização de enfermagem especializada e treinada, que é prática usual no atendimento das sobreviventes nos Estados Unidos, Inglaterra, Canadá e Austrália, com comprovação de significativa contribuição para atingir os objetivos dos programas criados para esse fim ${ }^{12,13,15,18}$. Higa et al. ${ }^{19}$ não mencionam a questão médico-pericial.

O modelo Bem-me-Quer passará por ampliação e contará com mais quatro centros de atendimento ${ }^{25,27}$ : Hospital Guilherme Álvaro, em Santos; Hospital Geral de Guarulhos; Hospital das Clínicas de Ribeirão Preto e Hospital das Clínicas de São José do Rio Preto.

Apesar de o Brasil ter despertado para a gravidade do problema há menos tempo que outros países, que em geral estudam e trabalham no assunto desde a década de oitenta, o Projeto Bem-Me-Quer já atende as diretrizes da OMS e dos demais protocolos conhecidos que tratam do tema. Este atendimento, porém, está concentrado em São Paulo e o grande desafio brasileiro é estendêlo a todas as regiões do país. Isto exigirá investimentos de vulto, em instalações, formação e capacitação de profissionais; grande envolvimento governamental, com o desenvolvimento de ações e programas articulados, envolvendo várias instâncias, tais como os Ministérios da Saúde, da Justiça e da Educação.

\section{CONCLUSÕES}

Os protocolos e iniciativas analisados são bastante semelhantes, porém destacam-se os dos Estados Unidos, da Inglaterra e da Índia por serem mais detalhados e ressaltarem a oferta simultânea de cunho assistencial, médico-legal e jurídico-policial no que for aplicável.

Nos Estados Unidos e na Inglaterra, além de protocolos bem estruturados, há ênfase no treinamento e educação continuada de equipes especializadas. A 
conjugação desses fatores aumenta a acurácia do processo pericial médico-legal, contribuindo para as boas práticas e a consequente identificação e condenação de agressores.

Finalmente, considerando-se as semelhanças entre extensão territorial, densidade populacional e estágio similar de mercado emergente, conclui-se que o Brasil poderia utilizar, inicialmente, a experiência da Índia como paradigma e, em seguida, da vivência dos Estados Unidos e da Inglaterra para implantar um protocolo próprio e de abrangência em todo o território nacional, como é desejável, desde que sejam disponibilizados os recursos financeiros e humanos.

Bonnet FR; Cintra RB. Protocols and initiatives for medico-legal assistance in sexual assaults cases against women: a comparison of initiatives in Brazil and abroad. . Saúde, Ética \& Justiça. 2014;19(1);45-51.

\begin{abstract}
Recent studies regarding sexual violence, particularly those related to assaults against women, report a high incidence of the phenomenon, ubiquitous in all countries, cultures, and social and economic levels. These studies have revealed that, in general, sexual assaults are rarely reported to law enforcement, and most will never be successfully prosecuted. This problem has been a long-standing source of frustration for survivors, lawyers of the victims, as well as members of the criminal justice systems of countries around the world. Based on a review of scientific studies, normative documents and practice reports from countries in five continents, this study aims to compare protocols and initiatives for medico-legal assistance in cases of sexual assaults against women, identifying the main gaps and challenges in Brazil, in order to improve forensic interventions and increase reporting and prosecution rates.
\end{abstract}

KEYWORDS: Sexual Violence; Forensic Medicine; Protocols; Brazil; National Strategies; Regional Health Planning.

\title{
REFERÊNCIAS
}

1.'Drezett J, Junqueira L, Antonio IP, Campos FS, Leal MCP, Iannetta R. Contribuição ao estudo do abuso sexual contra a adolescente: uma perspectiva de saúde sexual e reprodutiva e de violação de direitos humanos. Adolescência e Saúde. 2004; 1(4):31-9.

2. 'Du Mont J, White D. The uses and impacts of medicolegal evidence in sexual assault cases: a global review. Department of Gender, Women and Health, World Health Organization; 2007.

3. 'Lopes IMRS, Gomes KR, Silva BB, Deus MCBR, Galvão ERCGN, Borba DC. Caracterização da violência sexual em mulheres atendidas no projeto Maria-Maria em TeresinaPI. Rev Bras Ginecol Obstet. 2004; 26(2):35-9. DOI: http:// dx.doi.org/10.1590/S0100-72032004000200005.

4. 'Brasil. Presidência da República. Casa Civil. Subchefia para Assuntos Jurídicos. Decreto-lei $\mathrm{n}^{0}$ 2.848, de 07 de dezembro de 1940. Código Penal. Diário Oficial da União. Rio de Janeiro, RJ. 1940 dez 31.

5. 'Brasil. Presidência da República. Casa Civil. Subchefia para Assuntos Jurídicos. Lei no 12.015, de 07 de agosto de 2009. Altera o Título VI da Parte Especial do Decreto-Lei no 2.848, de 7 de dezembro de 1940 - Código Penal, e o art. 10 da Lei no 8.072, de 25 de julho de 1990, que dispõe sobre os crimes hediondos, nos termos do inciso XLIII do art. 5o da Constituição Federal e revoga a Lei no 2.252 , de 1 o de julho de 1954, que trata de corrupção de menores. Diário Oficial da União. Brasília, DF. 2009 ago 07.

6. 'Brasil. Presidência da República. Casa Civil. Subchefia para Assuntos Jurídicos. Lei $\mathrm{n}^{\circ} 12.845$, de $1^{\circ}$ de agosto de 2013. Dispõe sobre o atendimento obrigatório e integral de pessoas em situação de violência sexual. Diário Oficial da União. Brasília, DF. 2013 ago 02.

7. 'Mota JC. Violência contra a mulher praticada pelo parceiro íntimo: estudo em um serviço de atenção especializado [dissertação]. Rio de Janeiro: Escola Nacional de Saúde
Pública, Fundação Oswaldo Cruz; 2004.

8. 'Vergara, SC. Metodologia do trabalho científico. $22^{a}$ ed. São Paulo: Cortez; 2002.

9. 'Bardin L. Análise de conteúdo. Lisboa: Edições 70; 2009.

10.'CEHAT - Centre for Enquiry into Health and Allied Themes. Manual for medical examination of sexual assault. Mumbai: SatamUdyog Ed; 2012.

11.'WHO - World Health Organization. Department of Gender, Women and Health, \& Department of Violence, Injury Prevention and Disability. Guidelines for medico-legal care for victims of sexual violence. [Internet] Geneva; 2003. [acesso em 2013 out 28] Disponível em: http://whqlibdoc. who.int/publications.

12.'NSW Department of Health. Review of forensic and medical services for victims of sexual assault and child abuse. Report 1 - A new approach. [Internet] 2007. [acesso em 2013 nov 07]. Disponível em: http://www0.health.nsw. gov.au/pubs/2007/pdf/fms_volume_1.pdf

13.'NSW Department of Health. Review of forensic and medical services for victims of sexual assault and child abuse. Report 2 - Background and supporting information. [Internet] 2007. [acesso em 2013 nov 07]. Disponível em: http://www0.health.nsw.gov.au/pubs/2007/pdf/fms volume_2.pdf.

14.'CONNSACS - Commission on the Standardization of the Collection of Evidence in Sexual Assault Investigations. State of Connecticut Technical Guidelines for Health Care Response to Victims of Sexual Assault. [Internet] 2013. Disponível em: http://examguidelines.connsacs. org/wp-content/uploads/2013/08/2013-EVIDENCEGUIDELINES-FINAL-DRAFT.pdf.

15.'European Commission Directorate-General for Justice. Comparing sexual assault interventions (COSAI). Final Report. [Internet]. 2013. Disponível em: www.cosai.eu.

16.' Du Mont J, White D. Barriers to the effective use of 
Bonnet FR; Cintra RB. Protocolos e iniciativas de atendimento médico-legal em casos de violência sexual em mulheres.

medico legal findings in sexual assault cases worldwide. Qual Health Res. 2013; 23(9):1228-39. DOI: http://dx.doi. org/10.1177/1049732313502396.

17.'South Africa. National Management Guidelines for Sexual Assault. [Internet] 2003. [acesso em 2013 nov 12]. Disponível em: http://www.cecinfo.org/customcontent/uploads/2012/12/SouthAfrica-Sexual-AssaultGuidelines-2003.pdf

18.'United States of America. United States Department of Justice. A national protocol for sexual assault medical forensic examinations: adults/adolescents. [Internet] 2013. [acesso em 2013 out 28]. Disponível em: https://www. ncjrs.gov/pdffiles1/ovw/241903.pdf

19.'Higa R, Mondaca ADCA, Reis MJ, Lopes MHBM. Atendimento à mulher vítima de violência sexual: protocolo de assistência de enfermagem. Rev Esc Enferm USP. 2008; 42(2):377-82. DOI: http://dx.doi.org/10.1590/ S0080-62342008000200023.

20.'Hospital Pérola Byington. Núcleo de Atenção Integral à Mulher em Situação de Violência Sexual (AVS). [Internet]. São Paulo, SP. [acesso em 2013 set 10]. Disponível em: http://www.hospitalperola.com.br/programa-bem-me-quer. php

21.'SINAN - Sistema de Informação de Agravos de Notificação - Ministério da Saúde / SVS. [Internet]. 2010-2013. [acesso em 2013 set 10]. Disponível em: http://dtr2004. saude.gov.br/sinanweb/tabnet/dh?sinannet/violencia/bases/ violebrnet.def

22.'Secretaria da Segurança Pública do Governo do Estado de São Paulo (SSP/SP). Estatísticas. Violência Contra as
Mulheres. Ocorrências Registradas de estupro consumado, estupro tentado e outros c/c/ dignidade sexual de janeiro de 2010 a março de 2013. [Internet]. Disponível em: http:// www.ssp.sp.gov.br/novaestatistica/ViolenciaMulher.aspx.

23.'Brasil. Ministério da Saúde. Secretaria de Atenção à Saúde. Departamento de Ações Programáticas Estratégicas. Área Técnica de Saúde da Mulher. Prevenção e tratamento dos agravos resultantes da violência sexual contra mulheres e adolescentes: norma técnica. $2^{\mathrm{a}}$ ed. Brasília: Ministério da Saúde; 2005.

24.'Brasil. Presidência da República. Casa Civil. Subchefia para Assuntos Jurídicos. Decreto $n^{0} 7.958$, de 13 de março de 2013. Estabelece diretrizes para o atendimento às vítimas de violência sexual pelos profissionais de segurança pública e da rede de atendimento do Sistema Único de Saúde. Diário Oficial da União. Brasília, DF; 2013 mar 13.

25.'CRM-SP - Conselho Regional de Medicina do Estado de São Paulo. Saúde da mulher: Rede de assistência a vítimas de violência é ampliada em SP. [Internet]. 2012. Disponível em: http://www.cremesp.org.br/?siteAcao=Jornal\&id=1667.

26.'MPSP - Ministério Público do Estado de São Paulo. MP vai buscar convênio com hospital para compartilhar dados sobre abuso sexual. [Internet] 2012. [acesso em 2013 out 22]. Disponível em: http://www.mpsp.mp.br/portal/page/ portal/noticias/publicacao_noticias/2012/outubro_2012

27.'Diário Comércio Indústria \& Serviços. São Paulo recebe quatro centros de combate à violência contra a mulher. [Internet]. 2012. Disponível em: http://www.dci.com.br/ politica/sao-paulo-recebe-quatro-centros-de-combate-aviolencia-contra-a-mulher-id314857.html. 